\title{
Médiévales
}

Langues, Textes, Histoire

44 | printemps 2003

Le diable en procès

\section{Le Malleus Maleficarum à la lumière de l'historiographie : un Kulturkampf?}

The Malleus maleficarum and its Historiography: a Kulturkampf?

\section{Carmen Rob-Santer}

Traducteur : Benoît Grévin

\section{OpenEdition \\ Journals}

\section{Édition électronique}

URL : https://journals.openedition.org/medievales/732

DOI : 10.4000/medievales.732

ISSN : $1777-5892$

\section{Éditeur}

Presses universitaires de Vincennes

\section{Édition imprimée}

Date de publication : 1 juin 2003

Pagination : 155-172

ISBN : 2-84292-142-9

ISSN : 0751-2708

\section{Référence électronique}

Carmen Rob-Santer, «Le Malleus Maleficarum à la lumière de l'historiographie : un Kulturkampf?», Médiévales [En ligne], 44 | printemps 2003, mis en ligne le 03 novembre 2010, consulté le 23 avril 2022. URL : http://journals.openedition.org/medievales/732 ; DOI : https://doi.org/10.4000/medievales.732

Ce document a été généré automatiquement le 23 avril 2022.

Tous droits réservés 


\title{
Le Malleus Maleficarum à la lumière de l'historiographie : un Kulturkampf?
}

The Malleus maleficarum and its Historiography: a Kulturkampf?

\author{
Carmen Rob-Santer
}

Traduction : Benoît Grévin

\section{NOTE DE L'ÉDITEUR}

Traduit de l'allemand par Benoît Grévin

"Les frontières de ma langue sont les frontières de

mon monde."

Ludwig Wittgenstein

1 Le monde de la recherche relative au Malleus maleficarum se limite à peu près à deux langues : le latin, dans lequel le traité fut édité une trentaine de fois entre 1486 et 1669 , et l'allemand, dans lequel a été écrite la majeure partie - et de loin - du travail de recherche, et qui est aussi la langue dans laquelle a paru la première traduction complète $^{1}$ (œuvre de J.W. Richard Schmidt) en $1906^{2}$. Celle-ci - en dépit de son caractère fautif $^{3}$ - stimula, à travers ses nombreuses rééditions, l'intérêt pour le Malleus dans l'espace linguistique allemand. Le Malleus y reçut un statut exceptionnel, bien au-delà des frontières du monde scientifique, et fut désigné comme «le manuel de la chasse aux sorcières par excellence $»^{4}$. Jusqu'à la récente traduction de Wolfgang Behringer, Günter Jerouschek et Werner Tschacher ${ }^{5}$, c'est la version de Schmidt qui a servi de base aux nombreuses confrontations, même scientifiques, avec le Malleus.

2 Pourtant, cette large diffusion du Malleus contraste avec des incertitudes parfois profondes relatives à l'œuvre. On manque d'informations de base certaines, et le mauvais état de la recherche peut étonner. En particulier, des questions encore irrésolues concernant le rédacteur et l'importance du Malleus maleficarum rendent 
difficile un survol concis. Depuis les Lumières, le Malleus maleficarum oscille entre ces deux pôles de connaissance et de méconnaissance.

\section{L'état de la recherche}

\section{Le rédacteur et l'œuvre}

Le Malleus maleficarum, connu également sous le nom de Marteau des Sorcières [Hexenhammer] ${ }^{6}$ depuis le XVIII ${ }^{e}$ siècle, fut imprimé pour la première fois en $1486^{7}$ par Peter Drach, à Bâles. Le titre de l'ouvrage se déduit uniquement de l'apologie qui précède le traité publié par le prieur du couvent dominicain de Cologne, Jakob Sprenger. Cette apologie désigne comme responsable de la composition le collègue de Sprenger, le dominicain de Sélestat Heinrich Institoris (Kramer). Cela contredit néanmoins plusieurs passages du traité lui-même, dans lequel on parle de deux inquisiteurs en tant que rédacteurs. L'attribution à Kramer n'est pas discutée de son vivant, alors que le rôle de Sprenger reste obscur.

C'est dans l'édition de Nuremberg de 1519 qu'on trouve pour la première fois dans le titre une indication d'auteur, nommant aussi bien Institoris (mort avant 1505) que Sprenger (mort en 1495). Les éditions suivantes ne donnent pas d'auteur, les deux éditions vénitiennes de 1574 et 1576 attribuent l'ouvrage à Sprenger seul, tout comme la première édition de Francfort-sur-le-Main en 1580. Dans les éditions plus récentes de 1582, 1588 et 1600, le même imprimeur, en l'occurrence l'éditeur Nicolaus Bassaeus, mentionne deux auteurs. Comme c'est ordinairement le cas dans les éditions à partir de 1580, la dernière édition (Lyon, 1669) regroupe sous le nom de Malleus maleficarum plusieurs traités démonologiques, principalement ceux de Jean Nider, Bernard Basin, Girolamo Menghi, Ulrich Molitoris, Jean Gerson, Thomas Murner et Bartolomeo de Spina ; les auteurs du Marteau des Sorcières n'apparaissent pas dans la page de titre, mais sont donnés à la page suivante comme Iacobus Sprenger et Henricus Institoris 9 .

Jusqu'au XIXe siècle, Sprenger était considéré comme l'unique ou principal auteur. Joseph Hansen avança le premier de nombreux arguments pour une attribution principale ou unique à Institoris, déterminant ainsi la direction de la recherche en langue allemande $d u x^{e}$ siècle ${ }^{10}$, non sans de solides arguments ${ }^{11}$. En revanche, Sprenger continue à figurer dans la littérature internationale, au moins comme corédacteur à part entière. Ce n'est que récemment qu'André Schnyder tenta d'ébranler cette communis opinio allemande et laissa résolument ouverte la question de la coattribution à Sprenger ${ }^{12}$. Le débat sur l'attribution resta alors en suspens ${ }^{13}$.

Deux approbations de respectivement quatre et huit professeurs en théologie de l'université de Cologne ont été ajoutées au Malleus maleficarum au fil des éditions. Concernant la question de leur authenticité, c'est le verdict de Joseph Hansen sur la fausseté de la seconde qui a été suivi ${ }^{14}$. André Schnyder a avancé une contreproposition un siècle plus tard, et tenté de "décharger" les auteurs du Malleus de l'accusation de faussaire ${ }^{15}$.

7 On ajouta encore la bulle du pape Innocent VIII Summis desiderantes affectibus du 5 décembre 1484 aux éditions du Malleus ${ }^{16}$. D'une part, elle revêtait le Marteau des Sorcières d'un caractère d'autorité, et d'autre part, elle recevait, grâce à la publication 
commune, une place à part dans les promulgations papales concernant la sorcellerie et la magie.

\section{Forme, contenu et priorités du Malleus}

8 D'un point de vue formel, le Malleus maleficarum est divisé en trois parties. La première se compose de 18 questiones concernant l'origine de la sorcellerie, les rapports entre les démons et les sorcières, leurs pouvoirs et la question de la permission divine. Dans cette partie essentiellement théorique, le Marteau des Sorcières suit au plus près la méthode scolastique. C'est en première ligne la Bible et les écrits patristiques qui servent d'autorité, ainsi que les grands théologiens médiévaux. La seconde partie se compose de deux grandes questions principales : la première, en seize capitula, traite la question de savoir à qui le sorcier peut nuire ; la seconde, en neuf chapitres, expose les moyens de défense et de préservation face aux ensorcellements. Cette partie est étayée par l'expérience des deux inquisiteurs dans leur pratique des procès. La troisième partie propose des instructions pour la conduite des procès de sorcellerie. Une questio introductoria traite de la compétence juridique dans les procès de sorcellerie. Elle est suivie tout d'abord par une discussion en cinq questions sur l'ouverture du procès, puis par douze questions sur son déroulement, enfin par vingt questions sur les sentences applicables en fonction des cas.

9 Alors que, dans la première partie du Malleus, les explications théoriques sur la sorcellerie sont au premier plan, la seconde est caractérisée par un large emploi d'exempla, et la troisième est consacrée aux applications pratiques. À ces contenus variés semble correspondre chaque fois un public différent: la première partie s'adresse avant tout aux théologiens et leur procure les arguments nécessaires à la discussion, la seconde donne aux prédicateurs de nombreux exempla pour la prédication, et la troisième offre aux juristes un manuel de procédure complet.

10 Dans l'apologie, l'ouvrage est appelé opus novum simul et antiquum: antiquum certe materia et auctoritate; novum vero potius compilatione earumque aggregatione ${ }^{17}$. Pourtant, le Malleus maleficarum se différencie de ses prédécesseurs (et partiellement de ses successeurs) essentiellement dans les choix de ses priorités fondamentales. Deux caractéristiques de l'ouvrage sont déjà indiquées dans le titre : la focalisation sur les femmes en tant que sorcières (maleficarum, au lieu de maleficorum), ainsi que le maléfice (maleficium) comme objet digne de poursuite. De plus, le Malleus considérait la sorcellerie comme un crimen mixti fori, en conséquence de quoi non seulement la justice spirituelle, mais aussi temporelle, étaient incitées à réprimer les sorcières ${ }^{18}$.

11 La constitution de la sorcellerie en crime laïc se reflète essentiellement dans certaines insistances du Marteau des Sorcières ${ }^{19}$. En premier lieu doit être évoquée la prééminence du maleficium face aux autres composantes du concept générique de sorcellerie, qui permettait de justifier que les justices temporelles pussent juger du crime de sorcellerie. La dimension hérétique est alors relativisée ${ }^{20}$. Le Marteau des Sorcières laisse le plus souvent de côté les moments collectifs qui placeraient justement la sorcellerie dans la proximité d'une conjuration hérétique et des poursuites inquisitoriales: le sabbat des sorcières est à peine mentionné ${ }^{1}$, les actes sexuels avec des incubes n'ont lieu que dans le domaine privé, et non lors des assemblées de sorcières. Le moment du maleficium individuel, fondateur du mal, se révèle par contraste comme le motif idéal, pour en appeler à la justice séculière. 


\section{L'importance du Malleus}

12 "Il n'est pratiquement pas une publication concernant le Malleus qui omette de souligner l'influence exceptionnelle qu'aurait eue ce livre $»^{22}$. Dans la mesure où des critères peuvent être donnés à l'appui de cette assertion, ils consistent principalement dans le très grand nombre d'éditions, et leur durée. Pourtant, depuis une première présentation synthétique des éditions par Hain ${ }^{23}$, l'article de Joseph Hansen de $1898^{24}$ et les études de Amand Danet ${ }^{25}$, de nouvelles enquêtes font défaut. Les derniers résultats dans ce domaine ont été apportés par André Schnyder. Il oscille pourtant sans justification entre vingt-six ${ }^{26}$ et vingt-huit ${ }^{27}$ éditions pour les années 1487 à 1669.

13 On doit se demander rétrospectivement si ces critères pourraient être suffisants pour affirmer la place prééminente du Malleus Maleficarum dans la littérature sur la sorcellerie, même si on lui associe le qualificatif de « Manuel de la chasse aux sorcières par excellence ». On ressent cruellement le besoin de créer un catalogue de critères qui permettraient de prouver empiriquement cette assertion.

La réception du Marteau des Sorcières doit être considérée sous deux angles majeurs: littéraire et procédural ${ }^{28}$. Les informations sur l'histoire de la réception du Malleus maleficarum se retrouvent souvent isolément et hors contexte dans des enquêtes qui n'ont pas de rapport direct avec le Marteau des Sorcières ${ }^{29}$. On ne dispose pratiquement d'aucun élément concernant des réexploitations en littérature ou histoire des idées ; la démonologie semble encore être un peu «lugubre " pour l'histoire et l'histoire de la littérature allemandes ${ }^{30}$.

15 Concernant la réception du Malleus dans l'histoire de la procédure, on trouve des éléments avant tout dans des études d'histoire régionales concernant la chasse aux sorcières; au vu de l'accroissement des connaissances ponctuelles, une synthèse qui mette en perspective le Marteau des Sorcières serait hautement souhaitable.

Les historiens du droit semblent manifester depuis quelque temps un intérêt renforcé pour le thème ${ }^{31}$. Dans sa thèse Hexerei im Spiegel der Rechtstheorie («La sorcellerie au miroir des théories juridiques »), Michael Siefener livre par exemple, à travers de nombreux renvois au Malleus maleficarum, des données essentielles sur sa réception, et explicite certains critères déterminants pour l'importance d'un ouvrage ${ }^{32}$. Même si Michael Siefener ne qualifie pas le Malleus « d'ouvrage central de la sorcellerie » dans sa conclusion, il justifie très explicitement le poids qu'il donne au Malleus, parce qu'« on y renvoie en permanence dans la littérature secondaire pour son importance dans le combat contre les sorciers $»^{33}$.

Ces informations de base auront sans doute fait comprendre à quel point toute enquête sur le Marteau des Sorcières est liée à l'histoire de la recherche antérieure. Le postulat de départ sur l'importance de l'ouvrage provient essentiellement de l'historiographie allemande sur la sorcellerie du XviII ${ }^{\mathrm{e}}$ siècle, qui fut reçue par-delà les représentations $\mathrm{du} \mathrm{XIX}^{\mathrm{e}}$ siècle finissant et du début du $\mathrm{XX}^{\mathrm{e}}$ siècle. Le discours, dans lequel la position particulière $\mathrm{du}$ Malleus tient fréquemment lieu de paradigme, se révèle encore dominant dans la recherche allemande, à l'encontre de recherches plus récentes, qui ont relativisé à plusieurs reprises la signification du Marteau des Sorcières dans l'histoire. Par là se révèle la nécessité d'une réélaboration de l'historiographie relative au Malleus maleficarum. 


\section{Aspects de l'historiographie} scripta magica jouirent d'un grand retentissement, le Malleus, dans les années trente du XVIII ${ }^{e}$ siècle, est considéré comme "ce livre, sur lequel fut fondé [...] le savoir de base concernant la magie et les procès qui furent menés sous cette accusation", et «en vertu duquel [...] quelques centaines de milliers d'hommes perdirent leur honneur, leurs biens et leur vie $[. ..] »^{41}$. L'ouvrage de Hauber apparaît encore imprégné de l'ambiance des procès de sorcellerie.

Peu de temps après le dernier procès pour sorcellerie européen, celui de la servante Anna Göldin, dans le canton suisse réformé de Glaris, en l'an 1782, le pasteur évangélique Johann Moriz Schwager publia un Essai d'une histoire des procès de sorcellerie (Versuch einer Geschichte der Hexenprozesse) en 1784, qu'il dédia à Joseph II, le « vainqueur 
de la superstition et des tyrans de la conscience ». La présentation de Schwager est motivée par ses expériences de prédicateur concernant la superstition, qui « continue encore à nuire à la religion $»^{42}$, aussi bien que par sa croyance en un progrès continuel de la connaissance ${ }^{43}$. Sa conception de l'histoire directrice suit la pensée cicéronienne de l'historia magistra: " l'histoire est une maitresse d'enseignement pour les sages et un fouet pour les fous [...]. La superstition [...] est encore bien présente [...]. On doit donc lui opposer une histoire fidèle de la superstition et de ses funestes suites, qui agira et effraiera plus efficacement que des réfutations abstraites $»^{44}$. Sur plus de cent-soixantedix pages, on trouve " un choix d'extraits du Marteau des Sorcières et des informations détaillées sur ce livre maudit ${ }^{45}$, car $"$ les principes qui y sont contenus ont encore pour une part leurs amis et leurs défenseurs, quoique la plupart de nos théologiens ne connaissent même plus cette source répugnante $»^{46}$.

Dans sa Dämonomagie (1818), le pasteur et conseiller d'église de Lindheim Georg Conrad Horst, en opposition avec ses prédécesseurs, veut écrire " pleinement avec une absence de parti-pris ecclésiastique », car «la différence des églises ou des confessions [...] n'était jusqu'à présent que trop visible, en particulier, dans la présentation des procès de sorcellerie $\aleph^{47}$. Les ouvrages de Horst sont portés par la conscience de vivre dans une époque postérieure aux procès de sorcellerie, et il le doit au siècle des Lumières qui vient de $s^{\prime}$ écouler ${ }^{48}$. Lui aussi entretient un intérêt particulier pour les procès de sorcellerie, mais c'est le Marteau des Sorcières qu'il place désormais au premier plan : "On ne peut écrire sur les procès de sorcellerie, sans considérer ce livre [...] et même livrer un abrégé fidèle de ce livre [...] pour écrire l'histoire même des procès de sorcellerie $\aleph^{49}$. En raison de cette importance, Horst donne un abrégé de plus de soixante-quinze pages du Malleus. Autant Horst s'efforce à une présentation impartiale d'Innocent VIII et de sa bulle citée, autant on en rencontre moins de traces lorsqu'il s'agit du Marteau des Sorcières ${ }^{50}$.

Un des plus influents et également des plus anciens travaux encore saisissables dans les recherches récentes relatives au Marteau des Sorcières - tout au moins dans ses réélaborations - est l'Histoire des procès de sorcellerie (Geschichte der Hexenprozesse) du théologien luthérien et député au Landtag Wilhelm Soldan. Avec l'ouvrage de Soldan, qui parut pour la première fois en 1843, puis parut en 1880, dans une édition revue par son beau-fils Heinrich Heppe, et enfin pour la troisième fois dans une réélaboration de Max Bauer en 1911, c'est une synthèse fondamentale sur la croyance aux sorcières et les procès de sorcellerie en Europe qui était créée, et sur laquelle des générations entières de chercheurs purent s'appuyer, et s'appuyaient il y a peu de temps encore. William Monter fait dériver de la tendance rationaliste, qui imprègne la présentation de Soldan et des travaux de ses nombreux successeurs, le " paradigme de Soldan ${ }^{51}$.

La croyance au progrès du XIXe siècle se manifeste dans l'œuvre de Soldan, aussi bien que la distance envers l'Église romaine, qui avait été la première à mettre en forme doctrinale les croyances concernant la magie, sur la base de la littérature en langue latine. L'intérêt prédominant pour les "auteurs", ainsi que la focalisation sur une culture érudite et doctrinaire, qui trouva finalement son application dans la pratique juridique, caractérisent l'orientation primaire de l'écriture de l'histoire des sorcières au XIX ${ }^{\mathrm{e}}$ siècle.

Si Soldan part déjà en guerre contre le Malleus, et surtout contre l'Église romaine, avec le bagage conceptuel des Lumières, muni d'une plume acérée et des armes de la rhétorique, la tendance antiromaine devient particulièrement claire dans la seconde 
édition de 1880, retravaillée par le professeur de théologie protestante Heinrich Heppe. Elle porte indiscutablement la marque du Kulturkampf, qui couvait depuis les années 1830 dans les pays de langue allemande ${ }^{52}$, s'enflamma en 1868 en Autriche, et, après la fondation de l'Empire allemand en 1871, se déchaîna particulièrement dans la Prusse protestante, puissance hégémonique de l'Empire «petit-allemand $»^{53}$. Le Kulturkampf " profita » de la condamnation du libéralisme et du rationalisme dans le syllabus (1864) comme de la dogmatisation de l'infaillibilité papale (1870) par Pie IX, et aboutit à un combat de l'État et des esprits libéraux contre la politique catholique et l'Église. Le $\mathrm{XIX}^{\mathrm{e}}$ siècle fut traversé par le « combat de principes entre l'idée libérale de l'État et de la société, qui reposait sur les Lumières, et également, en Allemagne, sur le postulat d'une nation culturelle bourgeoise imprégnée de protestantisme ou postprotestantisme nordallemand et, d'un autre côté, le système traditionnel des normes et des valeurs que continuait à défendre, avant tout, l'Église catholique $~^{54}$. C'est dans cette tension qu'est née une grande partie de la plus ancienne histoire des sorcières ${ }^{55}$, qui était également l'occasion de mener des combats d'actualité. Mais comme les adversaires n'étaient pas nommés parfois et comme leurs points de vue n'étaient pas directement exposés, ils se dérobent à l'observateur contemporain, d'autant plus que les œuvres - et ainsi les positions - fort militantes et idéologiques ont disparu.

On peut observer ce poids croissant du Kulturkampf en comparant les éditions de Soldan et de Heppe. Ce n'est pas seulement la tendance à l'anticatholicisme, mais également la mise en valeur du Marteau des Sorcières pour la chasse aux sorcières qui sont renforcées ${ }^{56}$. La seconde édition de Geschichte der Hexenprozesse devient la base d'une recherche sur les sorcières florissante à la fin du XIx siècle, et laisse aussi des traces dans la recherche plus récente.

29 Aujourd'hui, on n'utilise plus guère que la troisième édition de 1911, qui est depuis peu à nouveau disponible en réédition ${ }^{57}$. Eu égard à la coloration idéologique dont on a esquissé l'histoire, l'éditeur, M. Bauer, ajoute à sa nouvelle édition : "La tendance à traiter de manière bénigne les persécuteurs protestants des sorcières, à l'encontre de ceux de l'ancienne Église, devait donc naturellement tomber $»^{58}$ - ce qui n'a pourtant pas changé grand chose à l'opposition au catholicisme.

À l'époque où le Kulturkampf de 1868 embrase l'Autriche, le professeur de théologie protestante viennois Gustav Roskoff publie son Histoire du diable (Geschichte des Teufels, 1869) en deux volumes, dans laquelle il consacre au "célèbre " ${ }^{59}$ Malleus maleficarum, seul traité de sorcellerie envisagé, un chapitre entier, et surtout un compte-rendu extensif. Roskoff croit « devoir dater la période de la poursuite juridique des sorcière de la parution de la bulle, en relation avec son commentaire pratique, le Marteau des Sorcières $"^{60}$.

31 L'Histoire du diable de Roskoff ne dévoilerait certes "pas d'empreinte confessionnelle, mais bien l'empreinte rationaliste et progressiste du protestantisme ${ }^{61}$. C'est contre l'une et l'autre que le prêtre et inspecteur de la commanderie de l'ordre teutonique, à Francfort-sur-le-Main, Johannes Diefenbach, veut lutter, avec son ouvrage La sorcellerie, avant et après la division confessionnelle en Allemagne (Der Hexenwahn vor und nach der Glaubensspaltung in Deutschland, 1886), dans lequel il expose une vision catholique militante de l'histoire des sorcières. Le livre se comprend avant tout comme une réfutation des erreurs et de la partialité de Soldan-Heppe, et revendique un caractère polémique, mais surtout apologétique ${ }^{62}$. Quant au Malleus: "Dans l'ensemble, le contenu de l'ouvrage n'est pas aussi mauvais que sa renommée $»^{63}$. 

et du Marteau des Sorcières, comme de la plus récente histoire catholique dans ce domaine ", c'est avec ce sous-titre que le pasteur évangélique Georg Längin publie en 1888 son ouvrage Religion et Procès de sorcellerie (Religion und Hexenprozess), qui est dirigé contre l'histoire de la sorcellerie catholique et en particulier contre Johannes Diefenbach. "Contre l'affirmation d'insignifiance et d'innocuité de la bulle et du Marteau des Sorcières, il nous a semblé nécessaire de présenter substantiellement le complexe d'idées dans lequel ces deux productions intellectuelles s'inscrivent $"^{64}$. Car, «si nous nous demandons quelles furent les causes de la diffusion des procès de sorcellerie dans le Saint Empire Romain Germanique, on rencontre la bulle sur les sorcières et le Marteau des Sorcières, ainsi que la foule des prêtres romains, excités par ces deux $»^{65}$.

Ammann publie en 1890, dans une frappante objectivité, les protocoles d'audition du procès de sorcellerie conduit en 1485 à Innsbruck par Institoris. Aurait été ajoutée à ces actes provenant des archives curiales de Brixen/Bressanone une notice marginale de vingt-deux pages «indiquant comment procéder dans l'introduction et le déroulement d'un procès de sorcellerie $»^{66}$. Hartmann Ammann publie en 1911 cette notice avec une très courte introduction, comme Travail préparatoire de Heinrich Institoris pour le « Malleus Maleficarum » (Eine Vorarbeit des Heinrich Institoris für den Malleus Maleficarum ${ }^{67}$. sur les procès de sorcellerie fut l'œuvre de Sigmund Riezler, avec son Histoire des procès de sorcellerie en Bavière (Geschichte der Hexenprozesse in Bayern, 1896). C'est de lui que provient le jugement sur le Malleus maleficarum le plus souvent repris, certainement à cause de son expressivité, comme «le livre à la fois le plus maudit et le plus niais, le plus insensé, mais aussi le plus nuisible de la littérature mondiale $\aleph^{68}$. Sigmund Riezler souligne particulièrement l'importance de la typographie pour la diffusion du Marteau des Sorcières ${ }^{69}$ et met en valeur le fait qu'il ne fut pas seulement reçu directement dans les cercles cultivés, mais agit aussi sur les masses populaires à travers "les prédications, les conversations, les images, la littérature populaire $»^{70}$.

Le Malleus maleficarum dut sa place exceptionnelle parmi les traités de sorcellerie avant tout aux travaux de l'archiviste de Cologne Joseph Hansen, qui jouissent encore aujourd'hui bien souvent d'une autorité sans restrictions au sein de la corporation historienne. Hansen remarque en 1889 dans son premier article qui annonce ses futurs travaux: «L'importance de l'ouvrage fatidique, [...] sa place dans le contexte de la sorcellerie et de la chasse aux sorcières, n'a pas encore été jusqu'alors exposée de manière scientifique ", même si jusqu'alors, dans le cadre des nombreuses enquêtes sur la sorcellerie, le Malleus avait été souvent mentionné- parfois en fonction d'orientations confessionnelles ${ }^{71}$.

Dans ses amples ouvrages Zauberwahn, Inquisition und Hexenprozess im Mittelalter (Sorcellerie, inquisition et procès de sorcellerie au Moyen Âge, 1900), comme dans la collection de matériel qui y est jointe, Sources et enquêtes sur l'histoire de la sorcellerie et de la chasse aux sorcières au Moyen Âge (Quellen und Untersuchungen zur Geschichte des Hexenwahns und der Hexenverfolgung im Mittelalter, 1901), Joseph Hansen se montre avant tout intéressé par le développement du "système de la sorcellerie ", qu'il décrit à partir d'une position rationaliste comme « un composé abstrus de fictions religieuses et populaires ». Il considère ce système comme complètement achevé en l'an 1480, donc 
avant la naissance du Malleus maleficarum ${ }^{72}$. Avec force rhétorique $^{73}$, Hansen livre également son jugement sur le Marteau des Sorcières, un « invraisemblable monstre plein de pestilence $»^{74}$. Joseph Hansen considère cette œuvre comme « une pierre angulaire dans l'histoire de la sorcellerie et de la persécution des sorcières ${ }^{75}$, et conclut à une influence importante, du fait de sa diffusion exceptionnellement large ${ }^{76}$.

Au-delà de ces travaux d'ensemble, Hansen s'est exprimé sur le Malleus maleficarum dans de volumineux articles, comme on l'a vu précédemment au sujet de l'approbation de Cologne $\mathrm{e}^{77}$. À ce sujet, un débat érudit au sujet de la fausseté ou de l'authenticité de cette expertise fait rage au début $\mathrm{du} \mathrm{xx}^{\mathrm{e}}$ siècle, entre Joseph Hansen, d'orientation libérale, et le théologien catholique proche de la société d'histoire allemande Görresgesellschaft Nikolaus Paulus. On y retrouve à nouveau des traces du Kulturkampf ${ }^{78}$. Hansen n'apporte pas de nouveaux arguments; il répète ses thèses, et se sert des arguments d'autorité et des attaques ad personam. La confrontation tient plus de la guerre de tranchée que de l'exploration de nouveaux champs de recherches. Même si certains arguments de Paulus semblent plausibles, c'est Hansen qui sort clairement vainqueur de la confrontation, si l'on prend l'histoire comme juge. Sa démonstration de la fausseté de l'imprimatur de Cologne vaut soit comme thèse définitive, soit comme position la plus probable dans la recherche, tandis que la position de Paulus reste largement négligée ${ }^{79}$.

À côté d'études sur le Malleus maleficarum ${ }^{80}$ et de la première traduction disponible ${ }^{81}$, le début $d u x^{e}$ siècle apporte également des enquêtes sur la vie et l'action de Heinrich Institoris, dont les activités dans le cadre de la chasse aux sorcières intéressent particulièrement la recherche ${ }^{82}$. Après ces publications à orientation biographique, la science (allemande) prend congé pour un assez long temps de toute recherche concernant spécifiquement le Malleus.

C'est avec la première traduction anglaise du Malleus maleficarum par Montague Summers $(1928)^{83}$ que le traité se diffuse dans l'espace linguistique anglo-saxon. Suivent les travaux de Henry Charles Lea, Materials Toward a History of Witchcraft, publiés pour la première fois en 1939 par Arthur C. Howland, qui font entre autres référence au contenu du Malleus comme aux recherches de Joseph Hansen.

Ce n'est que dans les années 1950 que le Marteau des Sorcières reprend à nouveau place dans une recherche de plus en plus internationale, dans laquelle les travaux de psychologie historique qui entrent en jeu apportent peu de connaissances nouvelles. Les travaux d'ensemble prédominants dans les années 1960 prouvent encore une fois la proximité des recherches sur le Malleus avec l'histoire de la sorcellerie en général, ce qui se reflète pleinement dans les paradigmes utilisés.

41 Le tournant paradigmatique dans l'histoire de la sorcellerie, à la fin des années 1960 et au début des années 1970 fait disparaitre les grandes conceptions unitaires concernant la sorcellerie, alors que le Malleus renforce sa présence dans des enquêtes ponctuelles depuis 1970. Ces dernières paraissent maintenant dans un joyeux multilinguisme et montrent pour partie un intérêt pour la démonologie. Tous ces travaux ne trouvent pourtant que peu de résonances immédiates dans la recherche sur le Marteau des Sorcières ${ }^{84}$. Les années 1980 s'écoulent sans travaux dignes d'intérêt concernant le Malleus maleficarum, jusqu'à ce que Peter Segl tente de donner une puissante impulsion à la recherche grâce à un colloque interdisciplinaire intitulé Marteau des Sorcières (Hexenhammer) en 1987. L'ouvrage collectif qui en est issu met néanmoins surtout l'accent sur le « contexte » du traitées. 

historique, on peut considérer le début des années 1990 comme un nouvelle étape dans les efforts de la recherche. En deux ans paraissent deux rééditions de la première édition du Malleus, dues à André Schnyder (1991) ${ }^{86}$ et Günter Jerouschek (1992) ${ }^{87}$. En 1993, André Schnyder livre un volume de commentaire de quelque cinq cent pages, qui propose des bases résolument neuves pour une exploitation du Malleus maleficarum ${ }^{88}$. Schnyder publie à côté une série d'articles sur le Malleus où prédominent d'abord les aspects historiques, ensuite les aspects littéraires. le Malleus maleficarum, également dans de gros travaux académiques, dont l'origine n'est depuis longtemps plus limitée à l'espace linguistique allemand. Des modèles d'explication du succès du Malleus maleficarum sont esquissés, de nouvelles approches sont testées ${ }^{89}$. La croyance aux sorcières n'est plus classifiée de manière générique comme « irrationnelle » (« Folie des sorcières », « Hexen-wahn »), mais lui est concédée une certaine logique interne en fonction d'approches fonctionnalistes ou systémiques ${ }^{90}$. Ici s'esquisse, depuis dix à quinze ans, un tournant presque révolutionnaire, dans lequel le concept directeur des Lumières, la raison, puis la rationalité, est remplacé, ou plutôt redéfini.

Malgré tout, en ce qui concerne le Marteau des Sorcières, un certain nombre de caractéristiques des travaux les plus récents peuvent être relevées : $1^{\circ}$ un manque de connaissance partiel de l'état de la recherche, qui se manifeste en particulier dans l'omission des rééditions du Malleus et la référence continue à la traduction de Schmidt ; $2^{\circ}$ la concentration de la recherche sur le Malleus dans l'espace linguistique allemand ; $3^{\circ}$ la négation continue des résultats de la recherche non-allemande, dans des travaux par ailleurs qualifiés; $4^{\circ}$ l'attachement sans remise en question à «l'importance déterminante» du Malleus; $5^{\circ}$ et enfin, la tendance à éveiller l'intérêt d'un public plus large par l'inclusion d'un « Malleus » dans le titre.

Le Malleus maleficarum se révèle à plusieurs reprises dans l'historiographie comme le terrain de lutte d'intérêts contradictoires, plutôt que comme un terrain d'enquête à proprement parler. S'il s'agissait à l'époque des Lumières d'une affirmation de la raison, dans la longue durée, c'est surtout comme théâtre de confrontations confessionnelles entre historiens de culture ecclésiastique qu'apparaît le Malleus au XIx siècle. Actuellement, c'est en particulier pour gagner la faveur du public que le Malleus semble être utilisé - et cela aussi dans la logique des congrès.

Entre les chercheurs des différents domaines linguistiques et de différentes orientations, aucune sorte de "combat» ni de confrontation n'a pourtant lieu. La prédominance historiquement fondée de la recherche allemande continue à s'entretenir elle-même. Les barrières linguistiques et les différences dans les perspectives de départ tracent encore aujourd'hui des frontières scientifiques. La prise de conscience de ces frontières pourrait représenter un premier pas vers leur dépassement. Un second pas consisterait à reconsidérer l'importance du Malleus maleficarum - largement infondée. Après tout, c'est de la différence que naît la connaissance. 


\section{NOTES}

1. Si les souhaits d'un des premiers chercheurs dans le domaine de la sorcellerie, Georg Conrad Horst, avaient été exaucés, le Malleus, qui est selon lui l'instrument fatal pour imposer la volonté de la bulle Summis desiderantes affectibus, n'aurait jamais été traduit : « Le Marteau des Sorcières n'a jamais été traduit, et ce fut fort sage et raisonnable. Si jamais, ce qui n'est guère à craindre, un écrivain ou un éditeur devait avoir cette idée folle, la police devrait intervenir ». Ce destin devait pourtant être épargné à Schmidt et ses successeurs. G. HoRST, Dämonomagie oder Geschichte des Glaubens an Zauberei und dämonische Wunder, mit besonderer Berücksichtigung desHexenprozesses seit den Zeiten Innocentius des Achten II, Francfort-sur-le-Main, 1818, p. 32 sq.

2. J. S CнміDт éd., Jakob Sprenger, Heinrich Institoris. Der Hexenhammer (Malleus Maleficarum), Munich, 1906.

3. Voir A.S CHNYDER, «Der Malleus maleficarum: Unvorgreifliche Überlegungen und Beobachtungen zum Problem der Textformen », dans P. SEGL éd., Der Hexenhammer. Entstehung und Umfeld des Malleus maleficarum von 1487, Cologne, 1988, p.127-149, sp. p. 142 sq. P. SEGL, Als die Ketzer fliegen lernten. Über den Hexen «wahn » im Mittelalter, Abensberg, 1991, p. 10-12. Un exemple frappant, repéré par l'œil vigilant de G. Jerouschek, devrait suffire : «Chez Schmidt (éd. 1983, p. 190) la promenade mentionnée comme solatii causa, c'est-à-dire une promenade digestive après le déjeuner, devient la " recherche d'une salade" ". G. JEROUSCHEK éd., Malleus maleficarum 1487 von Heinrich Kramer (Institoris). Faksimile der Handschrift von 1491 aus dem Staatsarchiv Nürnberg, Nr. D. 1521, Hildesheim-Zurich-New York, 1992, V.

4. P. SEGL, «Einführung des Herausgebers », dans P. SEGL éd., Der Hexenhammer, op. cit., n. 3, p. $1-16$, sp. p. 3.

5. W. Behringer, G. JerouscheK, W. TSCHACHER éd., Heinrich Kramer (Institoris). Der Hexenhammer. Malleus Maleficarum, Munich, 2000.

6. P. SEGL éd., op. cit., n. 3, p. 1. Hansen avait déjà souligné qu'Institoris lui-même aurait parlé de «Marteau des malfaiteurs" [Unholdenhammer]. Voir J. HANSEN, Quellen und Untersuchungen zur Geschichte des Hexenwahns und der Hexenverfolgung im Mittelalter, Bonn, 1901, p. 362 et 390.

7. Voir dernièrement W. Behringer, G. Jerouschek, W. Tschacher éd., op. cit., n. 5, p. 23-27.

8. G. JEROUSCHEK éd., op. cit., n. 3, p. XVf. A. SCHNYDER, Malleus Maleficarum von Heinrich Institoris (alias Kramer) unter Mithilfe Jakob Sprengers aufgrund der dämonologischen Tradition zusammengestellt. Kommentar zur Wiedergabe des Erstdruck von 1487 (Hain 9238), Göppingen, 1993, p. 432. A. SCHNYDER, «Der Malleus Maleficarum. Fragen und Beobachtungen zu seiner Druckgeschichte sowie zur Rezeption bei Bodin, Binsfeld und Delrio ", Archiv für Kulturgeschichte, 74, 1992, p. 323-338.

9. La page de titre elle-même: Indices auctorum, capitum, rerumque non desunt, indique, contrairement à W. Behringer, G. Jerouschek et W. Tschacher éd., op. cit., n. 5, p. 32, que les noms des auteurs du Marteau des Sorcières ne manquent pas.

10. H. A mmanN, «Eine Vorarbeit des Heinrich Institoris für den Malleus Maleficarum», Mitteilungen des Österreichischen Instituts für Geschichtsforschung, Ergänzungsband 8, 1911, p. 461-504. P. SEGL, « Heinrich Institoris. Persönlichkeit und literarisches Werk », dans P. SEGL éd., op. cit., n. 3, p. 103-126, sp. p. 116 sq. G. JEROUSCHEK éd., op. cit., n. 3, p. XII-XV.

11. J. HANSEN, op. cit., n. 6, p. 404-407.

12. A. Schnyder, Malleus... Kommentar, op. cit., n. 8, p. 419-422.

13. W. Behringer, G. Jerouschek, W. Tschacher éd., op. cit., n. 5, p. 31-69, se décident en fonction de nombreux indices pour Institoris; il reste cependant à prouver qu'une co-attribution à Sprenger doit être absolument écartée. 
14. J. HANSEN, "Der Malleus Maleficarum, seine Druckausgaben und die gefälschte Kölner Approbation vom J. 1487 », Westdeutsche Zeitschrift für Geschichte und Kunst, 17, 1898, p. 119-168, sp. p. 133-168. J. HANSEN, «Der Hexenhammer, seine Bedeutung und die gefälschte Kölner Approbatio vom Jahre 1487 », Westdeutsche Zeitschrift für Geschichte und Kunst, 26, 1907, p. 372-404, sp. p. 391-404.

15. A. Schnyder, Malleus... Kommentar, op. cit., n. 8, p. 422-425.

16. Ces quatre pièces furent imprimées après la première édition du Malleus maleficarum, si bien qu'elles furent reliées aux exemplaires encore invendus, ou lancées sur le marché comme éditions à part. G. JEROUSCHEK éd., op. cit., n. 3, p. XVI.

17. A. SCHNYDER éd., Malleus Maleficarum von Heinrich Institoris (alias Kramer) unter Mithilfe Jakob Sprengers aufgrund der dämonologischen Tradition zusammengestellt. Wiedergabe des Erstdrucks von 1487 (Hain 9328), Göppingen 1991, p. 2B.

18. Comme l'indique la déclaration d'intention du Malleus: "Cum principalis intentio nostra in hoc opere sit ab inquisitione maleficarum, quantum cum deo fieri posset, nos inquisitores partium superioris almanie exonerare suis iudicibus ad puniendum relinquendo. [...] Quare et presens opus aggressi sumus ». MM III, q. introductoria, A. Schnyder éd., op. cit., n. 17, p. 186B. $\mathrm{S}$. Leutenbauer s'est particulièrement intéressé à cette position à part du Malleus maleficarum dans la littérature, par rapport à la compétence juridique des inquisiteurs : Hexerei- und Zaubereidelikt in der Literatur von 1450 bis 1550. Mit Hinweisen auf die Praxis im Herzogtum Bayern, Berlin, 1972, p. 61-79.

19. Voir sur ce point M. SIEFENER, Hexerei im Spiegel der Rechtstheorie. Das crimen magiae in der Literatur von 1574 bis 1608, Francfort-sur-le-Main et al., 1992, p. 95, 107 sq., 133, 168 sq. et 172.

20. MM III, q. introductoria. A. SCHNYDER éd., op. cit., n. 8, p. 184C-185A.

21. MM I, 11 ; II ; 1, 2 ; 3. A. SCHNYDER éd., op. cit., n. 8, p. 64A, 96C, 97B-98B, 105A-B.

22. A. SCHNYDER, op. cit., n. 8, p. 323.

23. L. HAIN, Repertorium Bibliographicum, in quo libri ab arte typographica inventa usque ad annum MD. 2. 1, Stuttgart, Paris, 1831, p. 138 sq. (9238-9246).

24. J. HANSEN, « Der Malleus Maleficarum, seine Druckausgaben », loc. cit., n. 14, p. 122-133.

25. A. D anet, Henry Institoris (Kraemer). Jacques Sprenger. Le Marteau des Sorcières. Malleus Maleficarum. Traduit du latin et précédé de "L'Inquisiteur et ses sorcières », Grenoble, 1990, p. 16-19. Voir P. SEGL, loc. cit., n. 4, p. 2, n. 7.

26. A. SCHNYDER, op. cit., n. 17, p. 5.

27. A. SCHNYDER, Malleus... Kommentar, op. cit., n. 8, p. 2-33.

28. U. Falk souligne aussi le besoin d'enquêtes sur l'utilisation des modèles littéraires dans la pratique juridique, «Dem Leser zur Tortur ", Rechtshistorisches Journal, 12, 1993, p.119-130, sp. p. $127 \mathrm{sq}$.

29. Dernièrement W. BEHRINGER, « Heinrich Kramers "Hexenhammer" : Text und Kontext ", dans A. SCHMAUDER éd., Frühe Hexenverfolgung in Ravensburg und am Bodensee. Begleitband zur Tagung: der Hexenhammer von Heinrich Kramer und die frühe Hexenverfolgung in Ravensburg und Oberdeutschland, Constance, 2001, p. 83-124, sp. p. 84 : «Die Rezeptionsgeschichte des "Hexenhammers" steckt immer noch in den Anfängen. "

30. C'est ainsi que, par exemple, l'étude de S. Houdard, Les Sciences du diable. Quatre discours sur la sorcellerie (XVe-XVII s.), Paris, 1992, qui se situe entre la démonologie et la littérature sur les sorcières, et dans laquelle l'auteur confronte quatre traités de sorcellerie, dont le plus utilisé est le Malleus maleficarum, n'a pas encore eu d'écho dans la recherche sur le Malleus.

31. L'intérêt dominant en histoire du droit actuelle pour la genèse d'institutions encore opérantes aujourd'hui n'a pas précisément favorisé la recherche sur les chasses et les procès de sorcières.

32. M. SIEFENER, op. cit., n. 19. 
33. Ibid., p. 228.

34. Dans une argumentation de type historico-génétique (historisch-genetisches Argument), la genèse d'un fait historique se substitue à d'autres schémas explicatifs - souvent idéologiques.

35. W. BEHRINGER, « Neun Millionen Hexen. Entstehung, Tradition und Kritik eines populären Mythos ", Geschichte in Wissenschaft und Unterricht, 49, 1998, p. 664-685, sp. p. 664.

36. C. THOMAsius, Über die Hexenprozesse, éd. R. LIBERWIRTH, Weimar, 1967, p. 117.

37. Ibid., p. 159 : « weder die H. Schrift, noch die Römische und Päbstl. Rechte, oder die Gesetze der alten Francken, von solchen Hexen etwas gewust haben ».

38. Ibid., p. 169 : « Die Fabel von der Secte der Hexen ist a. 1400 in Italien entstanden. »

39. Ibid., p. 172 sq.

40. En ce qui concerne la responsabilité de la «bulle des sorcières » et du Marteau des Sorcières dans les procès de sorcellerie, on rencontre souvent la déduction d'un lien causal, dérivé de leur contemporanéité, c'est-à-dire sur la base d'une argumentation post hoc, ergo propter hoc : «La bulle et le Marteau des Sorcières, pour n'évoquer qu'eux, eurent également des conséquences pratiques immédiates. Alors qu'on sait peu de chose sur les chasses aux sorcières avant la bulle et le Marteau des Sorcières, elles se développent considérablement dès l'époque de leur parution ». G. LÄNGIN, Religion und Hexenprozess. Zur Würdigung des 400jährigen Jubiläums der Hexenbulle und des Hexenhammers sowie der neuesten katholischen Geschichtsschreibung auf diesem Gebiet, Leipzig, 1888, p. 77. Point de vue semblable dans S. RIEZLER, Geschichte der Hexenprozesse in Bayern. Im Lichte der allgemeinen Entwicklung dargestellt, Stuttgart, 1896 (réimp. Aalen 1968), p. 103.

41. E. Hauber, Bibliotheca, acta et scripta magica. Gründliche Nachrichten und Urtheile von solchen Büchern und Handlungen, welche die Macht des Teufels in leiblichen Dingen betreffen I, Lemgo, 1739², p. 50 sq. Hauber présenta la première liste d'éditions du Malleus maleficarum : Ibid., p. 39-41, sp. p. 91 et 319 .

42. J. SCHWAGER, Versuch einer Geschichte der Hexenprozesse, Berlin, 1784, p.11. Voir aussi ibid., p. 118.

43. Ibid., p. 14.

44. Ibid., p. 340

45. Ibid., p. 56.

46. Ibid., p. 103.

47. G. HORST, op. cit., n. 1, I, p. XV sq.

48. Ibid., II, p. 5 sq.

49. Ibid., II, p. 31.

50. Voir n. 1.

51. W. MONTER, "The Historiography of European Witchcraft : Progress and Prospects ", The Journal of Interdisciplinary History, 2, 1971/72, p. 435-45, sp. p. 436.

52. La première édition de Geschichte der Hexenprozesse de Soldan paraît peu après la fin des troubles de Cologne (1837-1841), pendant lesquels l'Église catholique résista victorieusement dans l'un de ses premiers conflits concrets avec l'État prussien, portant sur l'éducation religieuse des enfants issus de mariages mixtes.

53. On appelle petite-allemande [klein-deutsch] la solution politique adoptée en 1871 lors de la fondation du second Reich, constituant à rassembler les populations des États allemands en un Empire allemand en laissant de côté les populations allemandes de l'Empire autrichien, par opposition à la solution grande-allemande, qui aurait inclus la partie germanique de l'Autriche, et fut rejetée par Bismarck [n. d. t.].

54. R. LILL, «Einleitung: Kulturkämpfe im 19. Jahrhundert », dans R. LiLl éd., Der Kulturkampf, Paderborn, 1997, p. 11.

55. L'importance du Malleus maleficarum est abordée dans Geschichte der Hexenprozesse de Soldan avant tout du point de vue de la pratique juridique. 
56. Voir W. BEHRINGER, Hexen. Glaube, Verfolgung, Vermarktung, Munich, $2000^{2}$, p. 93.

57. W. SOldAN, H. HePPE, M. BAUER, Geschichte der Hexenprozesse, Cologne, 1999.

58. Ibid., p. XV.

59. G. RosKoff, Geschichte des Teufels, II, Leipzig, 1869, p. 225.

60. Ibid., p. 224.

61. J. DiefEnBACH, Der Hexenwahn vor und nach der Glaubensspaltung in Deutschland, Mayence, 1886, p. III.

62. Ibid., p. III $s q$.

63. Ibid., p. 224.

64. G. LÄNGIN, Religion und Hexenprozess, op. cit., p. V.

65. Ibid., p. 67.

66. H. AmmanN, « Der Innsbrucker Hexenprozess von 1845 », Zeitschrift des Ferdinandeums für Tirol und Vorarlberg, 34, 1890, p. 1-87, sp. p. 5.

67. H. A MMANN, «Eine Vorarbeit des Heinrich Institoris für den Malleus Maleficarum», Mitteilungen des Instituts für Österreichische Geschichtsforschung Suppl. 8., 1911, p. 461-505.

68. S. RIEZLER, op. cit., n. 41, p. 102 sq.

69. Ibid., p. 103.

70. Ibid., p. 129.

71. J. HANSEN, « Der Malleus Maleficarum, seine Druckausgaben », loc. cit., n. 14, p. 119.

72. J. HANSEN, Zauberwahn, Inquisition und Hexenprozess im Mittelalter, Bonn, 1901, p. 473.

73. La littérature destinée au grand public s'est ici appuyée sur la recherche historique la plus ancienne. La "rhétoricisation» (Rhetorisierung) de l'historiographie est aujourd'hui considérée par la recherche scientifique comme « une phase de développement pré-scientifique de la pensée historique ", J. Rüsen, Theorie der Geschichte, dans R. V. Dülmen éd., Das Fischer Lexikon Geschichte, Francfort-sur-le-Main, 1994, p. 32-52, sp. p. 38.

74. J. HANSEN, op. cit., n. 74, p. 474 sq. : « unglaublichen Monstrum voller Sumpfluft ».

75. Ibid., p. 498: «einen Angelpunkt in der Geschichte des Hexenwahns und der Hexenverfolgung ".

76. Ibid., p. 499.

77. Voir plus haut n. 14 .

78. N. Paulus, «Ist die Kölner Approbation des Hexenhammers eine Fälschung ? ", Historisches Jahrbuch, 28, 1907, p. 871-876. N. PAuLus, « Die Rolle der Frau in der Geschichte des Hexenwahns », Historisches Jahrbuch, 29, 1908, p.72-95, sp. p.75. J. H ANSEN, «Der Hexenhammer, seine Bedeutung ", loc. cit., n. 14, p. 373. Dans la recherche sur le Malleus, l'étendue de la séparation entre la littérature scientifique et la littérature destinée à un plus large public est remarquable. Un examen de l'argumentation de Hansen montre entre autres sa conviction personnelle que le contenu des travaux de Paulus «ne semblait pas devoir mériter de considération particulière » en raison de la forme de publication, conviction qui se modifia avec le changement de la forme de publication, alors même que le contenu restait (au mot près!) le même. N. PAulus, «Zur Kontroverse über den Hexenhammer », Historisches Jahrbuch, 29, 1908, p. 559-574.

79. Également A. SCHNYDER, Malleus... Kommentar, op. cit., n. 8, p. 422-425.

80. H. CROHNS, «Die Summa Theologica des Antonins von Florenz und die Schätzung des Weibes im Hexenhammer », Acta Societatis Scientiarum Fennicae 32.4, Helsingfors, 1906, p. 1-23.

81. J. SCHмiDT éd., op. cit., n. 2.

82. J. HANSEN, « Heinrich Institoris, der Verfasser des Hexenhammers und seine Tätigkeit an der Mosel im Jahre 1488 ", Westdeutsche Zeitschrift für Geschichte und Kunst, 26, 1907, p. 110-118. K. O. MÜLLER, «Heinrich Institoris, der Verfasser des Hexenhammers und seine Tätigkeit als Hexeninquisitor in Ravensburg 1484 », Württembergische Vierteljahresschrift für Landesgeschichte, 
19, 1910, p. 397-417. H. WiBEL, « Neues zu Heinrich Institoris », Mitteilungen des Instituts für Österreichische Geschichtsforschung, 34, 1913, p. 121-125.

83. M. SUMMERS, Malleus maleficarum. Translated with and introduction, bibliography and notes by the Rev. MS, Londres, 1928.

84. Font exception la traduction fondamentale pour l'espace francophone de A. Danet, op. cit., n. 25, en 1973, ainsi que l'article de S. Anglo, largement diffusé, «Evident Authority and Authoritative Evidence : The Malleus Maleficarum », dans S. Anglo éd., The Damned Art. Essays in the Literature of Witchcraft, Londres, 1977, p. 1-31.

85. P. SEGL éd., op. cit., n. 3.

86. A. SCHNYDER éd., op. cit., n. 17.

87. G. JEROUSCHEK éd., op. cit., n. 3.

88. A. SCHNYDER, Malleus... Kommentar, op. cit., n. 8.

89. Voir dernièrement P. BROEDEL, The Malleus Maleficarum and the Construction of Witchcraft: Encounters with the Supernatural between Theology and Popular Belief, Ann Arbor, 1998 (Diss. Washington 1998).

90. Voir ibid., p. 54. L'article de G. SCHWERHOFF, « Rationalität im Wahn. Zum gelehrten Diskurs über die Hexen in der frühen Neuzeit », Saeculum, 37, 1986, p. 45-82 ; sur le Malleus, p. 55-63.

\section{RÉSUMÉS}

À chaque fois que la sorcellerie est discutée dans la littérature historique, on voit nécessairement apparaître le Malleus maleficarum. Il est vrai que l'ouvrage connut près de trente éditions latines entre 1486 et 1669 et que les traductions modernes du xx siècle, en premier lieu celle en allemand de J.W.R. Schmidt, touchèrent un large public. Or, cette grande popularité contraste gravement avec le manque d'études sur le contexte précis et le contenu du livre. Cette situation exceptionnelle est peut-être due au fait que le traité a été publié avec la bulle pontificale Summis desiderantes affectibus, qui lui conféra une légitimité suprême. Mais les auteurs des Lumières, et surtout les polémistes de la période du Kulturkampf, ne résistèrent pas à l'opportunité qui leur était ainsi offerte de s'en prendre à l'« âge sombre » de l'Église catholique - bien qu'il soit prouvé que les Protestants aient été tout aussi actifs dans la persécution. L'instrumentalisation du livre a conféré aux discussions sur le Malleus maleficarum un caractère spécifiquement allemand. Il serait intéressant également d'adopter un regard critique non seulement sur l'importance du Malleus maleficarum dans les périodes postérieures, mais aussi d'analyser son contenu à l'intérieur des paradigmes intellectuels de son époque.

Whenever witchcraft is discussed in historical literature the Malleus maleficarum is bound to make its appearance. Its wide popularity - almost thirty Latin editions between 1486 and 1669 and the modern translations of the 20th century, above all the German one by J.W.R. Schmidt, aimed at a wider readership - stands in marked contrast to a lack of studies about the precise context and contents ot the book. The reason why it lent itself to such exceptional treatment can be found in the fact that it was published together with the papal bull Summis desiderantesaffectibus which served as an ultimate authorization of the treatise. In turn this proved to be an irresistible opportunity both for the authors of Enlightenment and, even more so, polemicists of Kulturkampf era to take a swipe at the "dark history" the Catholic Church - notwithstanding the 
fact that Protestants had proved to be at least as active in the persecution. This instrumentalization of the book served to give the discussions of the Malleus maleficarum a specifically German character. It might be interesting, however, to take a critical look not only at the actual importance of the Malleus maleficarum in later years, but also to analyse its contents within the intellectual paradigms of its period.

\section{INDEX}

Mots-clés : sorcellerie, Malleus maleficarum, historiography, H. Institoris, J. Sprenger Keywords : witchcraft, Malleus maleficarum, historiography, H. Institoris, J. Sprenger

\section{AUTEURS}

\section{CARMEN ROB-SANTER}

Cumberlandstrasse 61a/8 A-1140 Wien 